\title{
Third Annual Open Meeting of the UK Pharmacogenomics and Stratified Medicine Network Conference
}

\author{
Third Annual Open Meeting of the UK Pharmacogenetics and Stratified Medicine \\ Network \\ 14 January 2015, Wellcome Trust Genome Campus, Hinxton, Cambridge, UK
}

The third Annual Open Meeting of the UK Pharmacogenetics and Stratified Medicine Network was held on 14 January 2015 in association with the Wellcome Trust on the Wellcome Trust Genome Campus at Hinxton, Cambridge, UK. In the morning, speakers from Cancer Research UK, the Medical Research Council, Genomics England, Innovate UK (formerly TSB) and the Department of Health described the current major projects they are funding. In the afternoon, speakers from various universities around the United Kingdom presented data on pharmacogenetics and stratified medicine research covering diverse disease areas including cancers, warfarin dosing, Gaucher disease and rheumatoid arthritis.

Keywords: biomarkers $\bullet$ cancer $\bullet$ clinical trial design $\bullet$ personalized medicine

- pharmacogenetics $\bullet$ pharmacogenomics $\bullet$ precision medicine $\bullet$ stratified medicine

The third Annual Open Meeting of the UK Pharmacogenetics and Stratified Medicine Network was held on 14 January 2015 in association with the Wellcome Trust on the Wellcome Trust Genome Campus at Hinxton, Cambridge, UK. Delegates from a wide range of backgrounds including academia, industry and funding or regulatory bodies attended. The focus of this meeting was the research environment for pharmacogenetics and stratified medicine in the UK. Key themes included:

- Quality of experimental design and research infrastructure are crucial in pharmacogenetics trials;

- Depth of phenotyping is crucial in discovering and characterizing biomarkers;

- More integration and guidance is needed for the diagnostics development pathway;

- New diagnostic tests must be validated in real-life settings and optimized for economic viability;
- We are in the process of moving from single-gene tests to microarrays and gene panel tests, but the future is going to be multimodal: genomics, transcriptomics, proteomics, epigenomics and metabolomics.

\section{The UK funding environment}

- Jonathan Pearce from the Medical Research Council (MRC) emphasized the need for a less fragmented development pathway for diagnostics (as compared with therapies) and described the joint partnership between the Medical Research Council and the Engineering and Physical Sciences Research Council to support a network of up to eight interdisciplinary Molecular Pathology Nodes.

- Tony Soteriou from the Department of Health presented a selection of exciting research infrastructure developments funded by the National Institute for
Elizabeth H Bradshaw West Midlands Regional Genetics Laboratory, Birmingham Women's NHS Foundation Trust, Mindelsohn Way, Birmingham, UK

Tel.: +44121627 2710 ext. 6895 Elizabeth.Bradshaw@bwnft.nhs.uk 
Health Research (NIHR) including: the National BioSample Centre in Milton Keynes, which can store up to 20 million samples (with robotic retrieval) for use by National Health Service and academic researchers; the NIHR BioResource, which is a bank of thousands of volunteers who can be recalled for research into the links between genes and disease; the MRC-NIHR Phenome Centre, which is a metabolic phenotyping laboratory providing a range of NMR and MS assays as well as metabolomics training; and the Clinical Practice Research Datalink, which offers services related to data collection, methodology and running of interventional clinical trials.

- Loic Lhuillier described Innovate UK's (formerly TSB) Stratified Medicine Innovation Platform (SMIP), a $£ 200$ million 5-year program designed to speed up diagnostics development and encourage clinical uptake of stratified medicine in the UK. This will facilitate the testing of new diagnostics in real pathology lab settings. It will focus on cancer profiling and biomarker implementation (in particular inflammatory biomarkers).

\section{The UK social \& regulatory environment}

- Joyce Tait described Innogen's involvement in the TSB public dialogue on personalized medicine. She reported that the public were largely supportive of stratified medicine (although they preferred the term 'precision medicine'), so long as the mechanisms for granting access to medicines are fair and transparent.

- Sir Gordon Duff from St Hilda’s College (Oxford) explained the roles and structures of the National Institute for Biological Standards and Control (NIBSC) and the Medicines and Healthcare Products Regulatory Agency (MHRA), and in particular urged attendees to make use of the MHRA Innovation Office. This is a confidential service designed to help companies, SMEs and academics to navigate the regulatory process for new medicines, devices or approaches to their development and manufacture. He also described several tools for making the research process easier, including the TrialViz tool (for identifying suitable patients for recruitment to trials from GP/HES data) and the Early Access to Medicines Scheme (which protects physicians from liability for prescribing medicines off-label in areas of unmet need).

\section{Clinical trial design}

- Ian Walker from Cancer Research UK described several recent examples of Cancer Research
UK-funded pharmacogenomics research, including the METABRIC study which showed that breast cancer could be grouped into ten distinct clusters which were associated with particular outcomes [1]. He also described the Stratified Medicine Programme, of which the second part (Lung Matrix Trial) is now beginning. This trial represents a great step forward in the scale of how pharmacogenetics drug trials are conducted on all levels, in that, it is a truly national trial incorporating six drugs, 18 Experimental Cancer Medicine Centres and a 28-gene next-generation sequencing (NGS) panel. There will be 14 stratified arms initially but the flexible rolling protocol means that arms can be added or removed during the course of the trial depending on early results.

- Professor Tim Maughan from the University of Oxford spoke about improvements in complex biomarker clinical trial design and illustrated them with examples from his own work on colorectal cancer. He emphasized that patient understanding was greatly increased by providing the right information at the right time, for example by not overloading the patient with general information about a trial immediately after they have been given bad news. He also described the approach of giving patients a break in their chemotherapy early on, which did not harm the patient, during which time a drug versus placebo trial can be carried out. This gave the benefit of showing the effects of a drug at an early stage of disease, rather than at the end of life [2].

\section{Recent progress in research}

- Matthew Garnett described impressive highthroughput experiments performed at the Wellcome Trust Sanger Institute in which hundreds of cancer cell lines were screened for sensitivity to over a hundred drugs, with matching multimodal -omics data for each cell line (genome, transcriptome, methylome) [3]. However he warned that patients treated with targeted therapies often relapse within a few years, often due to mutations in the same pathway/loop as the original mutation.

- Ann Daly from Newcastle University reported on several pharmacogenetics success stories including: the use of CYP2C9/VKORC1 genotyping to guide warfarin dosing (EU-PACT) [4]; the use of CYP2C9 genotyping to predict poor metabolization of the prodrug of the antiplatelet agent clopidogrel (resulting in an FDA 'boxed warning' and 
corresponding gene test) [5,6]; the use of CYP2D6 genotyping to predict poor response to tamoxifen [7], through poor metabolization of tamoxifen to the active metabolite endoxifen; and the use of HLA genotyping to predict serious adverse drug reactions to abacavir and carbamazepine. Several of these are now available as gene tests either in a hospital setting or through direct-to-consumer services.

- Tim Cox from the University of Cambridge described strong competition between large companies to provide therapeutic enzymes for the niche group of approxiomately 300 patients with Gaucher disease (a rare single-gene lysosomal disorder causing skeletal disease, visceromegaly and neurological disease). Gaucher disease is particularly suitable for a stratified approach as it is clinically heterogeneous and genetically well characterized.

- Anne Barton from the University of Manchester described how stratified approaches could make it economically feasible to put a subset of rheumatoid arthritis patients onto expensive biological drugs during the critical 6 month window following initial diagnosis [8]. Data from several genome-wide association studies were inconclusive and suggested that response to anti-TNF therapy is likely to be polygenic [9-12]. However, several strong confounding factors were identified including psychological factors [13] and nonadherence to treatment regime [14].

- Tim Hubbard from Genomics England outlined the benefits of the 100,000 Genomes Project to the UK health system and economy [15]. The project will focus on common cancers, infectious diseases and rare inherited diseases where there is residual unmet need. A large amount of work has already gone into pilot studies investigating the infrastructure available for carrying out the work, including the capacity for extractions and biobanking as well as 'bake-off' competitions to identify the best sequencing and annotation providers.

In conclusion, this well-attended meeting was a timely and enjoyable mixture of talks spanning academic research, clinical trials, the funding environment and the UK regulatory context. In particular, it was a pleasure to hear about the implementation of several nationwide, multi-armed, flexibly-designed pharmacogenetics trials. It is reassuring to know that this work is strongly supported by funding bodies and that there are ways of smoothing the currently complex regulatory process.

The Pharmacogenetics and Stratified Medicine Network is expanding, visit the website [16] to join the network and benefit from regular events, funding information and our collaboration network. Abstracts and slides from the presentations are available on the website [17].

\section{Financial \& competing interests disclosure}

The author is a member of the UK Pharmacogenetics and Stratified Medicine Network. The author has no other relevant affiliations or financial involvement with any organization or entity with a financial interest in or financial conflict with the subject matter or materials discussed in the manuscript apart from those disclosed.

No writing assistance was utilized in the production of this manuscript.

\section{References}

1 Curtis C, Shah SP, Chin S-F et al. The genomic and transcriptomic architecture of 2,000 breast tumours reveals novel subgroups. Nature 486(7403), 346-352 (2012).

2 Maughan TS, Meade AM, Adams RA et al. A feasibility study testing four hypotheses with Phase II outcomes in advanced colorectal cancer (MRC FOCUS3): a model for randomised controlled trials in the era of personalised medicine? Br. J. Cancer 110(9), 2178-2186 (2014).

3 Garnett MJ, McDermott U. The evolving role of cancer cell line-based screens to define the impact of cancer genomes on drug response. Curr. Opin. Genet. Dev. 24, $114-119$ (2014).

4 Pirmohamed M, Burnside G, Eriksson N et al. A randomized trial of genotype-guided dosing of warfarin. N. Engl. J. Med. 369(24), 2294-2303 (2013).

determinants of response to clopidogrel and cardiovascular events. N. Engl. J. Med. 360(4), 363-375 (2009).

6 Shuldiner AR, O'Connell JR, Bliden KP et al. Association of cytochrome P450 2C19 genotype with the antiplatelet effect and clinical efficacy of clopidogrel therapy. JAMA 302(8), 849-857 (2009).

7 Province MA, Goetz MP, Brauch $\mathrm{H}$ et al. CYP2D6 genotype and adjuvant tamoxifen: meta-analysis of heterogeneous study populations. Clin. Pharmacol. Ther. 95(2), 216-227 (2014).

8 Farragher TM, Lunt M, Fu B, Bunn D, Symmons DPM. Early treatment with, and time receiving, first diseasemodifying antirheumatic drug predicts long-term function in patients with inflammatory polyarthritis. Ann. Rheum. Dis. 69(4), 689-695 (2010).

9 Plant D, Bowes J, Potter C et al. Genome-wide association study of genetic predictors of anti-tumor necrosis factor 
treatment efficacy in rheumatoid arthritis identifies associations with polymorphisms at seven loci. Arthritis Rheum. 63(3), 645-653 (2011).

10 Krintel SB, Palermo G, Johansen JS et al. Investigation of single nucleotide polymorphisms and biological pathways associated with response to TNF inhibitors in patients with rheumatoid arthritis. Pharmacogenet. Genomics 22(8), 577-589 (2012).

11 Mirkov MU, Cui J, Vermeulen SH et al. Genome-wide association analysis of anti-TNF drug response in rheumatoid arthritis patients. Ann. Rheum. Dis. 72(8), 1375-1381 (2013).

12 Cui J, Stahl EA, Saevarsdottir S et al. Genome-wide association study and gene expression analysis identifies CD84 as a predictor of response to etanercept therapy in rheumatoid arthritis. PLoS Genet. 9(3), e1003394 (2013).
13 Cordingley L, Prajapati R, Plant D et al. Impact of psychological factors on subjective disease activity assessments in patients with severe rheumatoid arthritis. Arthritis Care Res. 66(6), 861-868 (2014).

14 Bluett J, Morgan C, Thurston L et al. Impact of inadequate adherence on response to subcutaneously administered antitumour necrosis factor drugs: results from the Biologics in Rheumatoid Arthritis Genetics and Genomics Study Syndicate cohort. Rheumatol. Oxf. Engl. 54(3), 494-499 (2015).

15 The 100,000 Genomes Project. www.genomicsengland.co.uk/the-100000-genomes-project

16 The Pharmacogenetics and Stratified Medicine Network. www.uk-pgx-stratmed.co.uk

17 Third Annual Open Meeting of the UK Pharmacogenomics and Stratified Medicine Network Conference. www.uk-pgx-stratmed.co.uk 